\title{
Clinical indicators for severe prognosis of scrub typhus
}

This article was published in the following Dove Press journal:

Risk Management and Healthcare Policy

II October 2013

Number of times this article has been viewed

\section{Pamornsri Sriwongpan ${ }^{1,2}$ \\ Pornsuda Krittigamas ${ }^{3}$ \\ Pacharee Kantipong ${ }^{4}$ \\ Naowarat Kunyanone ${ }^{5}$ \\ Jayanton Patumanond'}

Sirianong Namwongprom ${ }^{1,6}$

'Clinical Epidemiology Program, Faculty of Medicine, Chiang Mai University, Chiang Mai, Thailand; ${ }^{2}$ Department of Social Medicine, Chiang Rai Prachanukroh Hospital, Chiang Rai, Thailand; ${ }^{3}$ Department of General Pediatrics, Nakornping Hospital, Chiang Mai, Thailand; ${ }^{4}$ Department of Internal Medicine, ${ }^{5}$ Department of Medical Technology, Chiang Rai Prachanukroh Hospital, Chiang Rai, Thailand; 'Department of Radiology, Faculty of Medicine, Chiang Mai University, Chiang Mai, Thailand
Correspondence: Sirianong Namwongprom Department of Radiology, Faculty of Medicine, Chiang Mai University, Chiang Mai 50200, Thailand

Tel +6653945458

Fax +6653945476

Email snamwong@med.cmu.ac.th
Background: The study explored clinical risk characteristics that may be used to forecast scrub typhus severity under routine clinical practices.

Methods: Retrospective data were collected from patients registered at two university-affiliated tertiary care hospitals in the north of Thailand, from 2004 to 2010. Key information was retrieved from in-patient records, out patient cards, laboratory reports and registers. Patients were classified into three severity groups: nonsevere, severe (those with at least one organ involvement), and deceased. Prognostic characteristics for scrub typhus severity were analyzed by a multivariable ordinal continuation ratio regression.

Results: A total of 526 patients were classified into nonsevere $(n=357)$, severe $(n=100)$, and deceased $(n=69)$. The significant multivariable prognostic characteristics for scrub typhus severity were increased body temperature (odds ratio $[\mathrm{OR}]=0.58,95 \%$ confidence interval $[\mathrm{CI}]=0.45-0.74, P<0.001)$, increased pulse rate $(\mathrm{OR}=1.03,95 \% \mathrm{CI}=1.01-1.05, P<0.001)$, presence of crepitation $(\mathrm{OR}=3.25,95 \% \mathrm{CI}=1.52-6.96, P=0.001)$, increased percentage of lymphocytes $(\mathrm{OR}=0.97,95 \% \mathrm{CI}=0.95-0.98, P=0.001)$, increased aspartate aminotransferase (every $10 \mathrm{IU} / \mathrm{L})(\mathrm{OR}=1.04,95 \% \mathrm{CI}=1.02-1.06, P<0.001)$, increased serum albumin $(\mathrm{OR}=0.47,95 \% \mathrm{CI}=0.27-0.80, P=0.001)$, increased serum creatinine $(\mathrm{OR}=1.83,95 \%$ $\mathrm{CI}=1.50-2.24, P<0.001)$, and increased levels of positive urine albumin $(\mathrm{OR}=1.43,95 \%$ $\mathrm{CI}=1.17-1.75, P<0.001)$.

Conclusion: Patients suspicious of scrub typhus with low body temperature, rapid pulse rate, presence of crepitation, low percentage of lymphocyte, low serum albumin, elevated aspartate aminotransferase, elevated serum creatinine, and positive urine albumin should be monitored closely for severity progression.

Keywords: severe scrub typhus, risk factors, rickettsial infection, complications

\section{Introduction}

Scrub typhus, an infectious disease caused by Orientia tsutsugamushi from chigger bites, is common in Asia-Pacific countries. ${ }^{1}$ In Thailand, there were 7,310 cases in 2011 and 9,000 cases in 2012, most prevalent in the northern region. ${ }^{2}$ An infected person may show only low grade fever, which disappears in a few days, or severe manifestations caused by complications of various organs in the second week. Pneumonia, acute respiratory distress syndrome, myocarditis, liver failure, acute renal failure, encephalitis, and shock from focal vasculitis and perivasculitis are some examples. Severe complications were reported in 2\%-36\% of cases and were associated with organ involvement, different serotype, and patient immunity. Severe complications may cause death, and mortality reports, which may be as high as $30 \%$ without proper treatment, varied from place to place..$^{3-10}$ 
An indirect immunofluorescence antibody test is the standard diagnostic test, but other methods, such as the indirect immunoperoxidase test and the polymerase chain reaction test, were developed later and are also used. However, these tests are still considered expensive in countries with limited health care resources. They require well-equipped laboratory settings, trained personnel, and may take many days to many weeks to provide results. ${ }^{1,11,12}$ The cheaper Weil-Felix test is, therefore, still used in the general hospitals of some countries; however, it has low sensitivity and specificity. ${ }^{7,12}$ The rapid immunochromatographic test is therefore used to help in making prompt treatment decisions in those countries, including Thailand. To make the test more specific, some countries developed their own tests utilizing the prevalent serotypes. ${ }^{12}$

In routine clinical practice, the disease is diagnosed from clinical signs and symptoms, the history of contact, and the initial laboratory tests in order to start treatment. Patients respond rapidly with early diagnosis and treatment, but when the diagnosis and treatment are delayed, complications are common and death may follow. ${ }^{3-5,13-15}$

Studies of risk factors for severe scrub typhus and/or death reported somewhat similar results: mainly they include abnormalities of laboratory findings such as leukocytosis, thrombocytopenia, hyperbilirubinemia, hypoalbuminemia, elevated transaminase, serum creatinine levels and abnormal chest X-ray. Nonlaboratory risk factors related to severity were headache, presence of eschar, and age more than 60 years. ${ }^{5,7,13}$

The present study aims to explore clinical risk characteristics that may be used to forecast disease severity under routine clinical practice. The findings may be incorporated into clinical evaluation, awareness, and prevention of disease complications, which may reduce case fatality.

\section{Material and methods}

\section{Patients}

Patients were those diagnosed with scrub typhus, registered in two university-affiliated tertiary care hospitals in Chiang Rai and Chiang Mai, in the north of Thailand, from 2004 to 2010. The guidelines for diagnosis used by the two hospitals followed the modified World Health Organization recommended surveillance standards (Table 1). ${ }^{9}$ Patients were categorized into three groups by their severity: (1) nonsevere, those without any complications; (2) severe, those with at least one organ involvement (Table 2); and (3) deceased, those who died of scrub typhus or complications following scrub typhus in the present admission. Patients were not included in the study if their discharge status was not stated, or if they were enrolled in another parallel study (a clinical trial on steroid treatment).
Table I Operational definitions of scrub typhus based on the World Health Organization

\begin{tabular}{lll}
\hline Patients with both & $\begin{array}{l}\text { Accompanied } \\
\text { by at least one }\end{array}$ & $\begin{array}{l}\text { Also accompanied } \\
\text { with at least one }\end{array}$ \\
\hline $\begin{array}{l}\text { Exposed to chiggers } \\
\text { (stayed or went into risk }\end{array}$ & $\begin{array}{l}\text { Headache } \\
\text { Myalgia }\end{array}$ & $\begin{array}{l}\text { Presence of eschar } \\
\text { Positive }\end{array}$ \\
$\begin{array}{l}\text { areas within } \\
\text { Profuse sweating }\end{array}$ & $\begin{array}{l}\text { immunochromatographic } \\
\text { of symptoms) }\end{array}$ & test for scrub typhus \\
Reported acute fever & Conjunctival & \\
(within 2 weeks of onset & Lymphadenopathy & \\
of symptoms) & Maculopapular rash & \\
\hline
\end{tabular}

Note: Copyright @ 2013. World Health Organization (WHO)2013. Reproduced with permissionWorldHealthOrganization.WHOrecommendedsurveillancestandards.2004: 123-I24. Availablefrom:http://www.who.int/csr/resources/publications/surveillance/ whocdscsrisr992.pdf. Accessed on July 21, $2013 .^{9}$

\section{Data collection}

Retrospective data were used, and key information was retrieved from in-patient medical files, out patient cards, laboratory reports and registers.

Table 2 Definitions of severe scrub typhus

\begin{tabular}{|c|c|}
\hline $\begin{array}{l}\text { System } \\
\text { involvement }\end{array}$ & haracteristics \\
\hline $\begin{array}{l}\text { Cardiovascular } \\
\text { system }\end{array}$ & $\begin{array}{l}\text { Presence of any of the following: } \\
\text { Systolic blood pressure }<90 \mathrm{mmHg} \\
\text { Abnormal cardiac arrhythmia with no previous } \\
\text { history of the following: } \\
\text { I. atrial fibrillation; } \\
\text { 2. supraventricular tachycardia; } \\
\text { 3. frequent premature ventricular tachycardia. } \\
\text { Myocarditis: elevated CK-MB above base line. }\end{array}$ \\
\hline $\begin{array}{l}\text { Respiratory } \\
\text { system }\end{array}$ & $\begin{array}{l}\text { Presence of acute respiratory distress syndrome, } \\
\text { defined as follows: } \\
\text { I. } \mathrm{PaO}_{2} / \mathrm{FiO}_{2}(\mathrm{mmHg})<200 \text { in room air; } \\
\text { 2. with bilateral interstitial infiltration on chest } \\
\text { X-ray; } \\
\text { 3. with normal cardio/thoracic ratio or no volume } \\
\text { overload of } \mathrm{CVP} \text { from central venous catheter. }\end{array}$ \\
\hline $\begin{array}{l}\text { Central } \\
\text { nervous system }\end{array}$ & $\begin{array}{l}\text { Presence of any of the following: } \\
\text { I. GCS } \leq 12 \text { without other causes; } \\
\text { 2. seizure without other causes; } \\
\text { 3. meningoencephalitis. }\end{array}$ \\
\hline Hematology & Platelet count $\leq 20,000 / \mathrm{mL}$ \\
\hline Urinary tract & $\begin{array}{l}\text { Presence of acute renal failure defined as } \\
\text { any of the following; } \\
\text { I. Creatinine } \geq 2 \mathrm{mg} / \mathrm{dL} \\
\text { 2. Creatinine change }>0.5 \mathrm{mg} / \mathrm{dL} / \mathrm{day}\end{array}$ \\
\hline $\begin{array}{l}\text { Gastrointestinal } \\
\text { tract }\end{array}$ & $\begin{array}{l}\text { Presence of hepatitis, as defined by the following: } \\
\text { I. elevated aspartate aminotransferase; } \\
\text { 2. or alanine aminotransferase more than five-fold } \\
\text { above baseline. }\end{array}$ \\
\hline
\end{tabular}

Note: Adapted from Chiang Rai Prachanukroh Hospital expert agreements on categorizing scrub typhus severity (with permission).

Abbreviations: CK-MB, myocardial muscle creatine kinase; CVP, central venous pressure; GCS, Glasgow Coma Scale; $\mathrm{PaO}_{2} / \mathrm{FiO}_{2}$, ratio of partial pressure arterial oxygen and fraction of inspired oxygen. 


\section{Study characteristics}

All of the following study characteristics were examined within the first day of admission:

- Demographic data: sex, age, underlying disease (diabetes, hypertension, chronic obstructive pulmonary disease, and liver cirrhosis).

- Clinical manifestations: headache, myalgia, cough, nausea, vomiting, abdominal pain, diarrhea, conjunctival injection, jaundice, eschar, maculopapular rash, lymphadenopathy, hepatomegaly, splenomegaly, stiff neck, seizure, crepitation, wheezing, and dyspnea.

- Vital signs: body temperature $\left({ }^{\circ} \mathrm{C}\right)$, systolic blood pressure $(\mathrm{mmHg})$, diastolic blood pressure $(\mathrm{mmHg})$, pulse (beats per minute), and respiration (breaths per minute).

- Laboratory findings: hematological tests, liver function tests, renal function tests, electrolyte profiles, urine albumin, and urine glucose.

\section{Data analysis}

The different characteristics across the three groups were tested by using nonparametric trend testing across ordered groups. The characteristics related to disease severity (presented with odds ratios [OR]) were explored and tested with multivariable ordinal continuation ratio logistic regression. Type 1 errors were set at $\leq 0.001$.

\section{Ethics statement}

The study was approved by Chiang Rai Prachanukroh Hospital Ethics Committee for Research, the Ethics Committee for Research, Nakornping Hospital, and the Ethical Committee on Research in Patients, Faculty of Medicine, Chiang Mai University. All traceable individual data were kept confidential throughout all processes of analysis.

\section{Results}

A total of 526 eligible cases of scrub typhus were classified into nonsevere $(n=357)$, severe $(n=100)$, and deceased $(n=69)$. They were similar in sex, underlying disease, myalgia, cough, nausea/vomiting, abdominal pain, conjunctival injection, eschar, maculopapular rash, hepatomegaly, splenomegaly, respiration rate, hematocrit, hemoglobin, globulin, potassium, and chloride (Tables 3 and 4).

Different characteristics across groups were age, headache, diarrhea, jaundice, lymphadenopathy, stiff neck, seizure, crepitation, wheezing, dyspnea, vital signs (body temperature, systolic blood pressure, diastolic blood pressure, pulse rate), hematological tests (white blood cell count, platelet count, neutrophils, lymphocytes, monocytes), liver function tests (aspartate aminotransferase [AST], alanine aminotransferase, alkaline phosphatase, total bilirubin, direct bilirubin, albumin), renal function test (blood urea nitrogen, creatinine), electrolyte profiles (sodium, carbon dioxide), and urine examinations (urine albumin, urine glucose) (Tables 3 and 4).

Under the multivariable ordinal continuation ratio logistic regression, the characteristics related to disease severity were increased body temperature $(\mathrm{OR}=0.58,95 \%$ confidence interval $[\mathrm{CI}]=0.45-0.74, P<0.001)$, increased pulse rate $(\mathrm{OR}=1.03,95 \% \mathrm{CI}=1.01-1.05, P<0.001)$, presence of crepitation $(\mathrm{OR}=3.25,95 \% \mathrm{CI}=1.52-6.96, P=0.001)$, increased percentage of lymphocytes (OR $=0.97,95 \%$ $\mathrm{CI}=0.95-0.98, P=0.001)$, increased AST (every $10 \mathrm{IU} / \mathrm{L}$ ) $(\mathrm{OR}=1.04,95 \% \mathrm{CI}=1.02-1.06, P<0.001)$, increased serum albumin $(\mathrm{OR}=0.47,95 \% \mathrm{CI}=0.27-0.80, P=0.001)$, increased serum creatinine $(\mathrm{OR}=1.83,95 \% \mathrm{CI}=1.50-2.24$, $P<0.001$ ), and increased levels of positive urine albumin $(\mathrm{OR}=1.43,95 \% \mathrm{CI}=1.17-1.75, P<0.001)($ Table 5) .

\section{Discussion}

The present study confirmed some clinical profiles related to scrub typhus severity.

\section{Vital signs}

The present study demonstrated that a decline of $1^{\circ} \mathrm{C}$, and a more rapid pulse rate, increased the risk of severe scrub typhus. Hypothermia (body temperature below $36^{\circ} \mathrm{C}$ ) and a pulse rate $>90$ beats per minute were consequences of systemic inflammatory response syndrome in patients with systemic infections, which led to septic shock in severe infection. ${ }^{16}$ Septic shock was reported as a common complication and risk of death in scrub typhus. ${ }^{3-5,17}$ In patients with septic shock, hypothermia was a significant risk factor for organ dysfunction and death, compared to those with fever. ${ }^{18}$

\section{Physical examinations}

The presence of crepitation was associated with scrub typhus severity in our study. Studies in the past all reported complications involving pulmonary systems, with $11 \%-54 \%$ of the cases resulting as a consequence of vasculitis and perivasculitis in the lungs. ${ }^{5,13,14,19-22}$ Chest X-rays showed interstitial infiltration, plural edema, pulmonary congestion, plural effusion, or cardiomegaly in $10 \%-64 \%$ of the cases. ${ }^{19-21,22}$ Patients may experience coughing or difficulty in breathing, and crepitation was reported in $23 \%-28 \%$ of 
Table 3 Demographic characteristics and clinical manifestations of patients with scrub typhus $(n=526)$

\begin{tabular}{|c|c|c|c|c|}
\hline Characteristics & $\begin{array}{l}\text { Nonsevere } \\
(\mathrm{n}=357)\end{array}$ & $\begin{array}{l}\text { Severe } \\
(n=100)\end{array}$ & $\begin{array}{l}\text { Deceased } \\
(n=69)\end{array}$ & $P$-value \\
\hline \multicolumn{5}{|l|}{ Demographics } \\
\hline Male & $201(56.3)$ & $55(55.0)$ & $35(50.7)$ & 0.413 \\
\hline Age (years) & $25.0 \pm 20.6$ & $32.6 \pm 21.8$ & $46.7 \pm 20.2$ & $<0.00$ I \\
\hline Underlying diseases & $30(8.4)$ & $14(14.0)$ & $8(11.6)$ & 0.186 \\
\hline \multicolumn{5}{|l|}{ Clinical manifestations } \\
\hline Headache & II 7 (32.8) & $29(29.0)$ & $14(20.3)$ & 0.041 \\
\hline Myalgia & $69(19.3)$ & $23(23.0)$ & I3 (I8.8) & 0.820 \\
\hline Cough & $120(33.6)$ & $32(32.0)$ & $17(24.6)$ & 0.172 \\
\hline Nausea, vomiting & $83(23.3)$ & $27(27.0)$ & $12(19.4)$ & 0.544 \\
\hline Abdominal pain & $118(33.1)$ & $35(35.0)$ & $25(36.2)$ & 0.565 \\
\hline Diarrhea & $60(16.8)$ & $25(25.0)$ & $17(24.6)$ & 0.047 \\
\hline Conjunctival injection & $330(92.4)$ & $90(90.0)$ & $64(92.8)$ & 0.823 \\
\hline Jaundice & II (3.I) & $21(21.0)$ & $17(24.6)$ & $<0.00$ I \\
\hline Eschar & $192(53.8)$ & $60(60.0)$ & $38(55.1)$ & 0.557 \\
\hline Maculopapular rash & $324(90.8)$ & $95(95.0)$ & $66(95.7)$ & 0.086 \\
\hline Lymphadenopathy & $237(66.4)$ & $73(73.0)$ & $63(91.0)$ & $<0.00$ I \\
\hline Hepatomegaly & II 3 (3I.7) & $36(36.0)$ & $14(20.3)$ & 0.198 \\
\hline Splenomegaly & $40(1 \mathrm{I} .2)$ & $9(9.0)$ & $4(5.8)$ & 0.157 \\
\hline Stiff neck & $6(1.7)$ & $4(4.0)$ & $4(5.8)$ & 0.032 \\
\hline Seizure & $4(1.1)$ & $10(10.0)$ & $12(17.4)$ & $<0.001$ \\
\hline Crepitation & I3 (3.6) & $18(18.0)$ & $25(36.2)$ & $<0.00$ I \\
\hline Wheezing & $2(0.6)$ & $6(6.0)$ & $7(10.1)$ & $<0.00$ I \\
\hline Dyspnea & $7(1.9)$ & $7(7.0)$ & $25(36.2)$ & $<0.00$ I \\
\hline \multicolumn{5}{|l|}{ Vital signs } \\
\hline Body temperature $\left({ }^{\circ} \mathrm{C}\right)$ & $38.5 \pm 1.1$ & $38.0 \pm 1.2$ & $37.5 \pm 0.9$ & $<0.00$ I \\
\hline SBP $(\mathrm{mmHg})$ & $105.8 \pm 14.1$ & $95.9 \pm 19.8$ & $99.9 \pm 23.9$ & $<0.00$ I \\
\hline $\mathrm{DBP}(\mathrm{mmHg})$ & $65.7 \pm 9.5$ & $58.8 \pm 13.6$ & $60.2 \pm 16.5$ & $<0.00$ I \\
\hline Pulse (beats/min) & $103 \pm 21.3$ & $104 \pm 22.5$ & $109.2 \pm 24.7$ & 0.049 \\
\hline Respiration (breaths/min) & $25.2 \pm 8.8$ & $27.4 \pm 10.7$ & $23.5 \pm 3.9$ & 0.058 \\
\hline
\end{tabular}

Notes: Values are $\mathrm{n}(\%)$ or mean $\pm \mathrm{SD}$; $P$-values are from nonparametric trend testing across ordered groups.

Abbreviations: DBP, diastolic blood pressure; SBP, systolic blood pressure; SD, standard deviation.

pediatric patients ${ }^{15,23}$ and $30.7 \%$ of adults. ${ }^{4}$ More severe pulmonary involvement may present with acute respiratory distress syndrome and death. ${ }^{4}$

\section{Percentage of lymphocytes}

All previous studies mentioned elevated white blood cell counts in severe scrub typhus, but there were no reports addressing the percentage of lymphocytes..$^{5,7,13,15}$ Some studies reported insignificant atypical lymphocytes in young scrub typhus patients. ${ }^{14,24} \mathrm{~A}$ study on the role of lymphocytes in scrub typhus reported early reduction of lymphocytes (mean \pm standard deviation $24.49 \% \pm 12.73 \%$ ), which was explained as the shifting of peripheral lymphocytes into the infected tissues. ${ }^{25}$ The present study also reported a significant effect of the percentage of lymphocyte reduction. It may be inferred that antibiotics might be indicated, even in the absence of lymphocytosis.

\section{Serum creatinine}

Serum creatinine indicated impaired kidney function. Renal failure manifested by elevated serum creatinine was commonly reported in scrub typhus and may be life threatening. ${ }^{3,7,17,22}$ A study from India also reported serum creatinine $>1.4 \mathrm{mg} \%$ as a risk predictor of death in scrub typhus. $^{7}$

\section{Aspartate aminotransferase}

Elevated AST reflects hepatocellular involvement in most infectious diseases. In scrub typhus, $80 \%$ of the patients experienced significantly elevated AST., ${ }^{3,7,17,26,27}$ A study from Thailand in young scrub typhus patients reported $96.3 \%$ of patients with high AST. In patients with hepatic dysfunction, AST levels were as high as $197.7 \pm 126.6 \mathrm{IU} / \mathrm{L} .{ }^{26}$ The present study reported elevated AST (every $10 \mathrm{IU} / \mathrm{L}$ ) as risk of severe scrub typhus. 
Table 4 Laboratory findings in patients with scrub typhus $(n=526)$

\begin{tabular}{|c|c|c|c|c|}
\hline Characteristics & $\begin{array}{l}\text { Nonsevere } \\
(n=357)\end{array}$ & $\begin{array}{l}\text { Severe } \\
(n=100)\end{array}$ & $\begin{array}{l}\text { Deceased } \\
(n=69)\end{array}$ & $P$-value \\
\hline \multicolumn{5}{|l|}{ Hematological } \\
\hline WBC $\left(\times 1000 / \mathrm{mm}^{3}\right)$ & $8.9 \pm 4.9$ & $11.4 \pm 5.4$ & $12.7 \pm 6.0$ & $<0.001$ \\
\hline Platelet $\left(\times 1000 / \mathrm{mm}^{3}\right)$ & $150.0 \pm 107.7$ & $111.9 \pm 110.7$ & $76.6 \pm 76.1$ & $<0.001$ \\
\hline Hematocrit (\%) & $35.2 \pm 6.3$ & $34.3 \pm 5.4$ & $36.2 \pm 6.8$ & 0.805 \\
\hline Hemoglobin (gm\%) & $11.9 \pm 2.1$ & $11.5 \pm 1.8$ & $12.1 \pm 2.3$ & 0.999 \\
\hline Neutrophil (\%) & $66.4 \pm 16.3$ & $77.3 \pm 15.0$ & $79.5 \pm 17.4$ & $<0.001$ \\
\hline Lymphocyte (\%) & $24.7 \pm 14.7$ & $14.7 \pm 11.7$ & $11.6 \pm 12.7$ & $<0.001$ \\
\hline Monocyte (\%) & $6.6 \pm 7.4$ & $5.0 \pm 8.4$ & $5.3 \pm 8.2$ & $<0.001$ \\
\hline \multicolumn{5}{|l|}{ Liver function } \\
\hline ALT (IU/L) & $94.5 \pm 94.5$ & $121.5 \pm 102.2$ & $253.7 \pm 606.8$ & 0.001 \\
\hline AST (IU/L) & $134.4 \pm 103.3$ & $207.6 \pm 164.6$ & $804.0 \pm 2,289.3$ & $<0.001$ \\
\hline ALP (IU/L) & $217.6 \pm 154.5$ & $333.1 \pm 273.5$ & $266.9 \pm 155.5$ & $<0.001$ \\
\hline Total bilirubin (mg/dL) & $1.3 \pm 1.7$ & $3.9 \pm 4.7$ & $5.9 \pm 7.9$ & $<0.001$ \\
\hline Direct bilirubin (mg/dL) & $0.5 \pm 1.0$ & $2.1 \pm 2.4$ & $3.4 \pm 3.9$ & $<0.001$ \\
\hline Albumin (g/dL) & $3.2 \pm 0.6$ & $2.7 \pm 0.5$ & $2.6 \pm 0.5$ & $<0.001$ \\
\hline Globulin (g/dL) & $3.3 \pm 0.7$ & $3.1 \pm 0.7$ & $3.3 \pm 0.7$ & 0.199 \\
\hline \multicolumn{5}{|l|}{ Renal function } \\
\hline BUN (mg/dL) & $15.5 \pm 12.1$ & $38.0 \pm 28.3$ & $55.0 \pm 34.0$ & $<0.001$ \\
\hline Creatinine (mg/dL) & $\mathrm{I} . \mathrm{I} \pm 0.8$ & $2.1 \pm 1.8$ & $3.5 \pm 3.1$ & $<0.001$ \\
\hline \multicolumn{5}{|l|}{ Electrolyte profile } \\
\hline $\mathrm{Na}(\mathrm{mmol} / \mathrm{L})$ & $133.8 \pm 3.9$ & $132.6 \pm 5.2$ & $132.6 \pm 6.1$ & 0.034 \\
\hline $\mathrm{K}(\mathrm{mmol} / \mathrm{L})$ & $3.5 \pm 0.5$ & $3.6 \pm 0.6$ & $3.8 \pm 0.9$ & 0.060 \\
\hline $\mathrm{Cl}_{2}(\mathrm{mmol} / \mathrm{L})$ & $10 I .1 \pm 4.6$ & $100.6 \pm 6.2$ & $100.0 \pm 7.7$ & 0.365 \\
\hline $\mathrm{CO}_{2}(\mathrm{mmol} / \mathrm{L})$ & $22.8 \pm 3.7$ & $20.2 \pm 4.5$ & $16.4 \pm 5.0$ & $<0.001$ \\
\hline \multicolumn{5}{|l|}{ Urine albumin } \\
\hline Negative & I $32(44.4)$ & $25(29.4)$ & $7(1.5)$ & $<0.001$ \\
\hline Trace & $58(19.5)$ & $15(17.7)$ & $5(8.2)$ & \\
\hline $1+$ & $49(16.5)$ & $17(20.0)$ & $10(16.4)$ & \\
\hline $2+$ & $45(15.2)$ & $24(28.2)$ & $20(32.8)$ & \\
\hline $3+$ & $13(4.4)$ & $4(4.7)$ & $16(28.2)$ & \\
\hline $4+$ & $0(0)$ & $0(0)$ & $3(4.9)$ & \\
\hline \multicolumn{5}{|l|}{ Urine glucose } \\
\hline Negative & $276(92.9)$ & 78 (9l.7) & 48 (78.7) & 0.002 \\
\hline Trace & $16(5.4)$ & $6(7.1)$ & II (18.0) & \\
\hline I+ & $\mathrm{I}(0.3)$ & $0(0)$ & I (I.6) & \\
\hline $2+$ & $\mathrm{I}(0.3)$ & $\mathrm{I}(\mathrm{I} .2)$ & $0(0)$ & \\
\hline $3+$ & $3(1.0)$ & $0(0)$ & $0(0)$ & \\
\hline $4+$ & $0(0)$ & $0(0)$ & $\mathrm{I}(\mathrm{I} .6)$ & \\
\hline
\end{tabular}

Notes: Values are $\mathrm{n}(\%)$ or mean $\pm \mathrm{SD}$. For urine albumin, trace is $5-20 \mathrm{mg} / \mathrm{dL} ; \mathrm{I}+, 30 \mathrm{mg} / \mathrm{dL} ; 2+, 100 \mathrm{mg} / \mathrm{dL} ; 3+, 300 \mathrm{mg} / \mathrm{dL} ; 4+, 2,000 \mathrm{mg} / \mathrm{dL}$. For urine glucose, trace is $100 \mathrm{mg} / \mathrm{dL} ; \mathrm{I}+, 250 \mathrm{mg} / \mathrm{dL} ; 2+, 500 \mathrm{mg} / \mathrm{dL} ; 3+, \mathrm{I}, 000 \mathrm{mg} / \mathrm{dL} ; 4+, 2,000 \mathrm{mg} / \mathrm{dL}$.

Abbreviations: ALP, alkaline phosphatase; $\mathrm{ALT}$, alanine aminotransferase; $\mathrm{AST}$, aspartate aminotransferase; $\mathrm{BUN}_{\mathrm{N}}$, blood urine nitrogen; $\mathrm{Cl}_{2}$, chloride; $\mathrm{CO}$, carbon dioxide; $\mathrm{K}$, potassium; $\mathrm{Na}$, sodium; SD, standard deviation; WBC, white blood cell.

\section{Serum albumin}

Patients with severe scrub typhus may have liver impairment causing a decline in albumin production. When associated with albumin leakages from blood vessels, as caused by vasculitis, patients may develop severe hypoalbuminemia. ${ }^{13,17,26}$ However, a previous study reported no differences in mortality among patients with different albumin levels. ${ }^{28}$ In our study, a decline in serum albumin, as measured in $\mathrm{g} / \mathrm{dL}$, increased the risk of more severe scrub typhus, even after excluding patients with liver cirrhosis (data not shown).

\section{Urine albumin}

The presence of albumin in urine indicated renal pathology. Renal vasculitis, which causes leakage of albumin into the urine, was reported in scrub typhus patients with renal complications. Acute renal failure may cause shock; a study 
Table 5 Multivariable analysis of prognostic indicators for scrub typhus severity $(n=526)$

\begin{tabular}{lll}
\hline Indicators & $\begin{array}{l}\text { Multivariable } \\
\text { odds ratio }(95 \% \mathrm{CI})\end{array}$ & P-value \\
\hline Body temperature $\left({ }^{\circ} \mathrm{C}\right)$ & $0.58(0.45-0.74)$ & $<0.00 \mathrm{I}$ \\
Pulse rate (beats/min) & $\mathrm{I} .03(\mathrm{I} .0 \mathrm{I}-\mathrm{I} .05)$ & $<0.00 \mathrm{I}$ \\
Crepitation & $3.25(\mathrm{I} .52-6.96)$ & $0.00 \mathrm{I}$ \\
Lymphocyte $(\%)$ & $0.97(0.95-0.98)$ & $0.00 \mathrm{I}$ \\
AST $(\times \mathrm{I0} \mathrm{IU/L)}$ & $\mathrm{I} .04(\mathrm{I} .02-\mathrm{I} .06)$ & $<0.00 \mathrm{I}$ \\
Serum albumin $(\mathrm{g} / \mathrm{dL})$ & $0.47(0.27-0.80)$ & $0.00 \mathrm{I}$ \\
Serum creatinine $(\mathrm{mg} / \mathrm{dL})$ & $\mathrm{I} .83(\mathrm{I} .50-2.24)$ & $<0.00 \mathrm{I}$ \\
Urine albumin* & $\mathrm{I} .43(\mathrm{I} .17-\mathrm{I} .75)$ & $<0.00 \mathrm{I}$ \\
\hline
\end{tabular}

Note: *Represents urine albumin levels from negative to $4+$.

Abbreviations: AST, aspartate aminotransferase; $\mathrm{Cl}$, confidence interval.

in Thailand reported septic shock in $88.9 \%$ of patients with positive urine albumin. ${ }^{17}$ In India, the corresponding figure was $28.6 \%$ in adults ${ }^{29}$ and $3 \%-17 \%$ in children. ${ }^{15,23}$ In the present study, higher levels of urine albumin significantly increased scrub typhus severity, even after excluding patients with diabetes, hypertension, and liver cirrhosis, all conditions in which positive urine albumin may already be present (data not shown).

The most important limitation of this report was the fact that the diagnosis of scrub typhus was based on routine practice as recommended by the World Health Organization for medical resource limited developing countries, where the indirect immunofluorescence antibody test, or more standard (and more complex) methods, were not readily available. Scrub typhus in this study, therefore, was actually suspected scrub typhus according to the World Health Organization's definition; however, we, the authors, believe that this is the more realistic situation currently occurring in poor developing countries.

\section{Conclusion}

Patients suspicious of scrub typhus with low body temperature, rapid pulse rate, crepitation, low percentage of lymphocytes, low serum albumin, elevated AST, elevated serum creatinine, and positive urine albumin may be at risk for more severe scrub typhus. Clinicians encountering such patients should be aware of disease progression to more severe states, and might consider early investigation or monitoring for systemic involvement to reduce or avoid further complications and death.

\section{Acknowledgment}

The authors wish to thank the authorities of Chiang Rai Prachanukroh Hospital and Nakornping Hospital for their support. The study was partially supported by a grant from The Graduate School, Chiang Mai University, Chiang Mai, Thailand.

\section{Disclosure}

The authors report no conflicts of interest in this work.

\section{References}

1. Watt G, Kantipong P. Orientia tsutsugamushi and scrub typhus. In: Ruoult D, Parola P, editors. Rickettsial Diseases. London, UK: Informa Healthcare; 2007:237-256.

2. Bureau of epidemiology; Ministry of public health; Thailand. Annual epidemiological surveillance report. 2012. Available from: http://www. boe.moph.go.th/Annual/Total_Annual.html. Accessed July 21, 2013.

3. Tsay RW, Chang FY. Serious complications in scrub typhus. J Microbiol Immunol Infect. 1998;31(4):240-244.

4. Chrispal A, Boorugu H, Gopinath KG, et al. Scrub typhus: an unrecognized threat in South India - clinical profile and predictors of mortality. Trop Doct. 2010;40(3):129-133.

5. Lee CS, Hwang JH, Lee HB, Kwon KS. Risk factors leading to fatal outcome in scrub typhus patients. Am J Trop Med Hyg. 2009;81(3): 484-488.

6. Suputtamongkol Y, Suttinont C, Niwatayakul K, et al. Epidemiology and clinical aspects of rickettsioses in Thailand. Ann N Y Acad Sci. 2009;1166:172-179.

7. Varghese GM, Abraham OC, Mathai D, et al. Scrub typhus among hospitalized patients with febrile illness in South India: magnitude and clinical predictors. $J$ Infect. 2006;52(1):56-60.

8. Wie SH, Chang UI, Kim HW, et al. Clinical features of 212 cases of scrub typhus in southern region of Gyeonggi-Do and the significance of initial simple chest $\mathrm{x}$-ray. Infect Chemother. 2008;40(1):40-45.

9. World Health Organization. WHO recommended surveillance standards. 2004:123-124. Available from: http://www.who.int/csr/ resources/publications/surveillance/whocdscsrisr992.pdf. Accessed on July 21, 2013.

10. Wang CC, Liu SF, Liu JW, Chung YH, Su MC, Lin MC. Acute respiratory distress syndrome in scrub typhus. Am J Trop Med Hyg. 2007;76(6): $1148-1152$.

11. Sonthayanon P, Chierakul W, Wuthiekanun V, et al. Rapid diagnosis of scrub typhus in rural Thailand using polymerase chain reaction. Am J Trop Med Hyg. 2006;75(6):1099-1102.

12. Koh GC, Maude RJ, Paris DH, Newton PN, Blacksell SD. Diagnosis of scrub typhus. Am J Trop Med Hyg. 2010;82(3):368-370.

13. Kim DM, Kim SW, Choi SH, Yun NR. Clinical and laboratory findings associated with severe scrub typhus. BMC Infect Dis. 2010;10:108.

14. Jim WT, Chiu NC, Chan WT, et al. Clinical manifestations, laboratory findings and complications of pediatric scrub typhus in eastern Taiwan. Pediatr Neonatol. 2009;50(3):96-101.

15. Kumar M, Krishnamurthy S, Delhikumar CG, Narayanan P, Biswal N, Srinivasan S. Scrub typhus in children at a tertiary hospital in southern India: clinical profile and complications. J Infect Public Health. 2012;5(1):82-88.

16. Levy MM, Fink MP, Marshall JC, et al; International Sepsis Definitions Conference. 2001 SCCM/ESICM/ACCP/ATS/SIS International Sepsis Definitions Conference. Intensive Care Med. 2003;29(4):530-538.

17. Thap LC, Supanaranond W, Treeprasertsuk S, Kitvatanachai S, Chinprasatsak S, Phonrat B. Septic shock secondary to scrub typhus: characteristics and complications. Southeast Asian J Trop Med Public Health. 2002;33(4):780-786.

18. Marik PE, Zaloga GP. Hypothermia and cytokines in septic shock. Norasept II Study Investigators. North American study of the safety and efficacy of murine monoclonal antibody to tumor necrosis factor for the treatment of septic shock. Intensive Care Med. 2000;26(6): $716-721$. 
19. Charoensak A, Chawalparit O, Suttinont C, et al. Scrub typhus: chest radiographic and clinical findings in 130 Thai patients. J Med Assoc Thai. 2006;89(5):600-607.

20. Chen HC, Chang HC, Chang YC, et al. Chest radiographic presentation in patients with scrub typhus. Trans R Soc Trop Med Hyg. 2012;106(1): 48-53.

21. Jeong YJ, Kim S, Wook YD, Lee JW, Kim KI, Lee SH. Scrub typhus: clinical, pathologic, and imaging findings. Radiographics. 2007;27(1): 161-172.

22. Wu KM, Wu ZW, Peng GQ, Wu JL, Lee SY. Radiologic pulmonary findings, clinical manifestations and serious complications in scrub typhus: experiences from a teaching hospital in eastern Taiwan. Int $J$ Gerontol. 2009;3(4):223-232.

23. Rajendran A. Scrub typhus in paediatric age group: a report from a tertiary care hospital. J Pediatr Sci. 2011;3(2):e82.

24. Sirisanthana V, Puthanakit T, Sirisanthana T. Epidemiologic, clinical and laboratory features of scrub typhus in thirty Thai children. Pediatr Infect Dis J. 2003;22(4):341-345.
25. Cho BA, Ko Y, Kim YS, et al. Phenotypic characterization of peripheral T cells and their dynamics in scrub typhus patients. PLoS Negl Trop Dis. 2012;6(8):e1789.

26. Chanta C, Triratanapa K, Ratanasirichup P, Mahaprom W. Hepatic dysfunction in pediatric scrub typhus: role of liver function test in diagnosis and marker of disease severity. J Med Assoc Thai. 2007; 90(11):2366-2369.

27. Hu ML, Liu JW, Wu KL, et al. Short report: abnormal liver function in scrub typhus. Am J Trop Med Hyg. 2005;73(4):667-668.

28. Lee CS, Min IS, Hwang JH, Kwon KS, Lee HB. Clinical significance of hypoalbuminemia in outcome of patients with scrub typhus. $B M C$ Infect Dis. 2010;10:216.

29. Attur R, Kuppasamy S, Bairy M, et al. Acute kidney injury in scrub typhus. Clin Exp Nephrol. Epub January 5, 2013.
Risk Management and Healthcare Policy

\section{Publish your work in this journal}

Risk Management and Healthcare Policy is an international, peerreviewed, open access journal focusing on all aspects of public health, policy, and preventative measures to promote good health and improve morbidity and mortality in the population. The journal welcomes submitted papers covering original research, basic science, clinical \& epidemio-

\section{Dovepress}

logical studies, reviews and evaluations, guidelines, expert opinion and commentary, case reports and extended reports. The manuscript management system is completely online and includes a very quick and fair peerreview system, which is all easy to use. Visit http://www.dovepress.com/ testimonials.php to read real quotes from published authors.

Submit your manuscript here: http://www.dovepress.com/risk-management-and-healthcare-policy-journal 